\title{
ARTES VISUALES, ENSEÑANZA Y PANDEMIA
}

VISUAL ARTS, TEACHING AND PANDEMIC

Pilar Marchiano

marchiano.pilar@gmail.com Instituto de Investigación en Producción y Enseñanza del Arte Argentino y Latinoamericano. Facultad de Artes Universidad Nacional de La Plata. Argentina

\section{Resumen}

Este artículo reflexiona acerca de la enseñanza de las Artes Visuales durante la pandemia del coronavirus y el consecuente aislamiento. La distancia de la praxis docente habitual permite tener una profunda vigilancia epistemológica: ¿a quiénes enseñamos?, ¿cuál es el sentido de la enseñanza?, ¿qué y cómo enseñamos? Este contexto de urgencia, en el cual se adoptó la virtualidad como herramienta educativa, nos plantea interrogantes sobre las relaciones de enseñanza-aprendizaje, la dinámica de las clases, la planificación, los objetivos, las consignas y la evaluación.

\section{Palabras clave}

Artes visuales; enseñanza; pandemia

\section{Abstract}

This article ponders on the teaching of the Visual Arts during the coronavirus pandemic and the quarantine. The distance from the usual teaching practice allows us to have a profound epistemological vigilance: who do we teach? What is the purpose of teaching? What do we teach? How do we teach? In this context of urgency, the virtuality was adopted as an educational tool. This raises questions about teaching-learning relationships, class dynamics, planning, objectives, instructions and evaluation.

\section{Keywords}

Visual arts; teaching; pandemic 
Escribo este artículo durante el aislamiento obligatorio por la pandemia del coronavirus. Un momento excepcional para el mundo en el cual se repiensan las relaciones sociales, la comunicación, la solidaridad, las desigualdades y, por supuesto, también la educación y el papel de la institución educativa. Por eso, estas palabras intentan conformar una suerte de bitácora o de memoria sobre la enseñanza de las Artes Visuales durante la pandemia.

Ante la incertidumbre abundan las charlas, las videoconferencias, los seminarios, las jornadas y los cursos virtuales enfocados en la educación en este contexto, que sirven de guía para las y los docentes, pero que también se convierten en espacios vinculares y de acompañamiento. Por consiguiente, el presente escrito contiene reflexiones abordadas tanto en estas instancias como en intercambios entre colegas que nos permiten hacer una profunda vigilancia epistemológica sobre nuestras prácticas. Como escribe Paulo Freire (2006), para una práctica docente crítica debemos ser conscientes de nuestra incompletud: uno no es, sino que está siendo.

\section{¿Queremos volver iguales?}

Se escucha que la normalidad que conocíamos ya no existe y que debemos prepararnos para enfrentar una nueva normalidad, ${ }^{1}$ en la cual la vida cotidiana, las relaciones sociales y la educación, tanto obligatoria como universitaria, no serán (de hecho, ya no son) iguales. ¿Cómo nos preparamos, entonces, las y los docentes para dar clases en este nuevo modo de estar siendo en el mundo? En este momento, en el que aún estamos en nuestras casas, resulta arriesgado dar una respuesta a este interrogante; sin embargo, podemos identificar algunos de los cambios generados por la pandemia en relación con la educación.

El aislamiento nos hace valorar la presencialidad, lo performático de las clases, la simultaneidad de los cuerpos, la comunicación sincrónica, la comunicación gestual, el aula heterogénea, la diversidad de voces, el debate e intercambio, el trabajo entre pares (tanto entre docentes como entre estudiantes). Inés Dussel (en Canal ISEP, 2020) hace referencia a la importancia de la escuela como un espacio otro, un espacio diferente al de los hogares, en el cual las y los estudiantes pueden desarrollar su autonomía. La escuela y la universidad son espacios de encuentro, de sociabilización y de intercambio; espacios en los que se genera un proceso de enseñanza y de aprendizaje colectivo.

El estar en el aula es irremplazable, no obstante debemos rastrear las herramientas para seguir enseñando. La virtualidad se convirtió en la herramienta central -mails, reuniones por Zoom o por Google Meet, videos de Youtube, Padlet, Google Drive, grupos de Facebook, Instagram, entre otros-, aunque es indiscutible que pone en evidencia y que acentúa las inequidades en la accesibilidad de recursos. 
La distancia nos quita muchas oportunidades de aprendizaje, pero nos permite ser críticos acerca de la normalidad anterior: ¿queremos volver iguales? Tenemos la opción de reflexionar acerca de qué queremos modificar, qué mantener y qué profundizar sobre el sistema educativo, las relaciones de enseñanza-aprendizaje, la dinámica de las clases, la formulación de las consignas y la evaluación.

\section{Claridad, síntesis y simplicidad}

La enseñanza de las Artes Visuales en la educación obligatoria arrastra consigo imaginarios relacionados con diferentes corrientes pedagógicas, entre ellos el de la expresión, vinculado a la corriente progresista o expresionista. En este modelo, el docente es un guía que interviene lo menos posible en la enseñanza artística con el fin de que las y los estudiantes puedan expresar, mediante la producción manual, su mundo interior. Si la función docente es la de no intervenir en el proceso creador, entonces el arte no se puede enseñar, es un don innato que se activa con la inspiración.

La pedagogía crítica, en la cual nos posicionamos, ha superado este modo de comprender a la educación artística, ya que entiende que el arte es un campo de conocimiento (Consejo Federal de Educación, 25 de agosto de 2010, 30 de septiembre de 2010, 15 de agosto de 2012) con contenidos específicos enseñables y que, por lo tanto, se aprenden. Sin embargo, el imaginario sigue arraigado y dificulta la enseñanza, ya que no solo está asentado, muchas veces, en las y los estudiantes, sino también en docentes de otras áreas y en las familias: «[...] la enseñanza de las Artes Visuales en la escolaridad obligatoria aún sigue ligada a las categorias de creatividad, de libertad, de innatismo o bien a imperativos aplicacionistas» (Belardinelli \& Catibiela, 2017, p. 84).

Durante las clases de Artes Visuales, en un contexto habitual de no pandemia, la intervención docente es primordial para afirmar el enfoque crítico y desmitificar ciertos imaginarios. Pero cuando la comunicación es asincrónica y el material que les llega a las y los estudiantes es a través de cuadernillos o de videos, hacer presente esta perspectiva se vuelve más dificultoso. ¿Qué sucede cuando la materia se reduce al momento de diversión sin una reflexión crítica sobre lo que se produce?, ¿hay un aprendizaje significativo y una construcción y apropiación de los conocimientos? En este sentido, es interesante recuperar las "palabras prohibidas» que propone Diana Aisenberg en su texto MDA. Apuntes para un aprendizaje del arte (2018). La autora prohíbe el uso de la palabra lúdico como sinónimo de divertido y espontáneo, ya que «los juegos son cosa seria» (Aisenberg, 2018, p. 53). No es la intención apartar la diversión del arte, y menos en este contexto en el que es necesario encontrar esos momentos, sin embargo la enseñanza de las Artes Visuales no debe limitarse a ellos. 
En la educación universitaria no hacemos frente a este imaginario de la enseñanza artística ligada solo a la expresión, la creatividad y la diversión, aunque no queda exceptuada de las problemáticas que puede traer la no presencialidad, principalmente en las materias proyectuales donde el espacio de taller es fundamental.

Por esta razón, tanto en la educación obligatoria como en la universitaria, hoy más que nunca, debe haber una claridad en la formulación de la consignas, en la planificación, en los objetivos, en las explicaciones, en las devoluciones y en la evaluación. Rebeca Anijovich (2016) destaca la importancia de las consignas auténticas y significativas, las cuales "al ser explícitas, estar escritas y contemplar actividades con sentido, contribuyen al desarrollo de la autonomía de los estudiantes» (p. 50). Además de la claridad, son fundamentales la síntesis y la simplicidad, las cuales no implican la pérdida de complejidad. "Lo que nosotros tenemos que hacer es lograr una simplicidad que no minimice la seriedad del objeto estudiado sino que la resalte» (Freire, 2006, p. 26).

La virtualidad dificulta y ralentiza la instancia en la cual las y los docentes conocemos los intereses de las y los estudiantes. Por esta razón, las consignas no solo deben contemplar la claridad, la síntesis y la simplicidad, sino también una apertura que permita múltiples posibilidades de resolución con el objetivo de que ellas y ellos plasmen su subjetividad.

En la charla «Cuarentena, evaluación y después» (en fba a la carta, 2020) dictada por Daniel Belinche, Graciana Pérez Lus y Silvia Andrea Cristian Ladaga, organizada por la Facultad de Artes (FDA) de la Universidad Nacional de La Plata (UNLP) durante los primeros meses de la cuarentena, se reflexionó acerca de la evaluación y de la diferencia que esta supone en el nivel universitario con respecto del nivel primario y secundario. En la educación universitaria se acreditan saberes, es decir, interesa que haya una coherencia entre el proceso y el resultado -relación más flexible en la educación obligatoria-. No obstante, hay veces en las que el proceso se diluye en los resultados de los exámenes parciales o finales. De este modo, el obstáculo que impone la virtualidad con respecto a estos exámenes se convierte en la oportunidad de reflexionar sobre estas instancias definitorias. Así, la evaluación, y la resultante calificación, se centran en la resolución de trabajos prácticos y en la participación en foros o en muros colaborativos, en los cuales no solo se trabajan los contenidos, sino que se abordan con mayor profundidad, ya que la construcción de los conocimientos se produce paulatinamente.

La evaluación formativa[... ] entiende al aprendizaje como un proceso que el alumno recorre a través de una variedad de actividades por medio de las cuales adquiere, estructura, organiza y reestructura sus conocimientos. El propósito principal de la evaluación formativa es mejorar los aprendizajes de los estudiantes y aumentar la probabilidad de que todos aprendan (Anijovich, 2016, p. 91). 
Asimismo, «la evaluación en proceso o continua permite considerar los reales avances en el dominio flexible del conocimiento artístico» (Consejo Federal de Educación, 15 de agosto de 2012). Por medio de la retroalimentación, la evaluación no es una tarea solo del docente, sino que las y los estudiantes pasan a tener un lugar central, ya que regulan su propio aprendizaje (Anijovich \& Mora, 2010).

\section{Enseñanza de las Artes Visuales}

El modelo tradicional de la educación establece un límite entre el interior y el exterior. Luego, la pedagogía crítica hace permeable ese límite y la vida cotidiana pasa a formar parte de la institución educativa. Ahora, esta relación se invirtió, ya que es la institución la que ingresa en los hogares. Por eso, más que nunca hay que tener en cuenta las subjetividades, los modos de vida, los recursos de cada familia y de cada estudiante.

La enseñanza de las Artes Visuales comenzó a recurrir a actividades que implican la utilización de los espacios del hogar y de los objetos que cada estudiante tiene en su casa. Como dice Francesco Tonucci (en Nicolás Trotta, 2020): «La casa puede ser un laboratorio» (16:26). Se recrean obras de arte famosas mediante la fotoperformance, se hacen instalaciones con objetos de uso cotidiano para crear diversidad de espacios, se construyen imágenes efímeras, se sacan fotografías y se intervienen digitalmente, entre muchas otras posibilidades. De este modo, se ponen en ejercicio la poetización de lo cotidiano y la transdisciplina, y se produce un acercamiento a las prácticas artísticas contemporáneas que muchas veces quedan fuera de las clases. Mientras que en el aula, principalmente en la educación obligatoria, suele ser una dificultad proponer actividades con materialidades diversas, en las casas las y los estudiantes las tienen a su disposición.

A través de las propuestas que les brindamos, proveemos acercamientos a los conocimientos y facilitamos experiencias, y, cuanto más diversas sean las experiencias, mayores serán las posibilidades de aprendizaje. En este sentido Gabriela Augustowsky (2012) retoma los postulados de John Dewey sobre la noción de experiencia en la enseñanza:

[...] concebir el arte como experiencia significa diseñar acciones, proyectos, propuestas en las que chicos, chicas y jóvenes sean incitados a ocupar la escena en un movimiento que los involucre personalmente, íntimamente; que los convoquen de modo genuino a la construcción de sentidos propios para repensarse individual y colectivamente a través del arte (p. 31).

La experiencia implica las subjetividades. Cada sujeto experimenta de maneras diferentes, por lo que la experiencia es democratizadora: «[...] todas las personas están en condiciones de participar de experiencias estéticas» (Augustowsky, 2012, p. 15). 
A modo de ejemplo, en cuarto grado del nivel primario trabajamos con objetos cotidianos con el propósito de estudiar la superposición y el gradiente y contraste de tamaño en el entorno [Figura 1]. Las y los estudiantes no solo plasmaron su subjetividad, sino que fue a través de la experiencia y del juego con los materiales que reflexionaron sobre las relaciones espaciales -que varian según el punto de vista-y sobre la construcción de nuevos espacios.

Figura 1. Trabajos realizados por estudiantes de cuarto grado de nivel primario

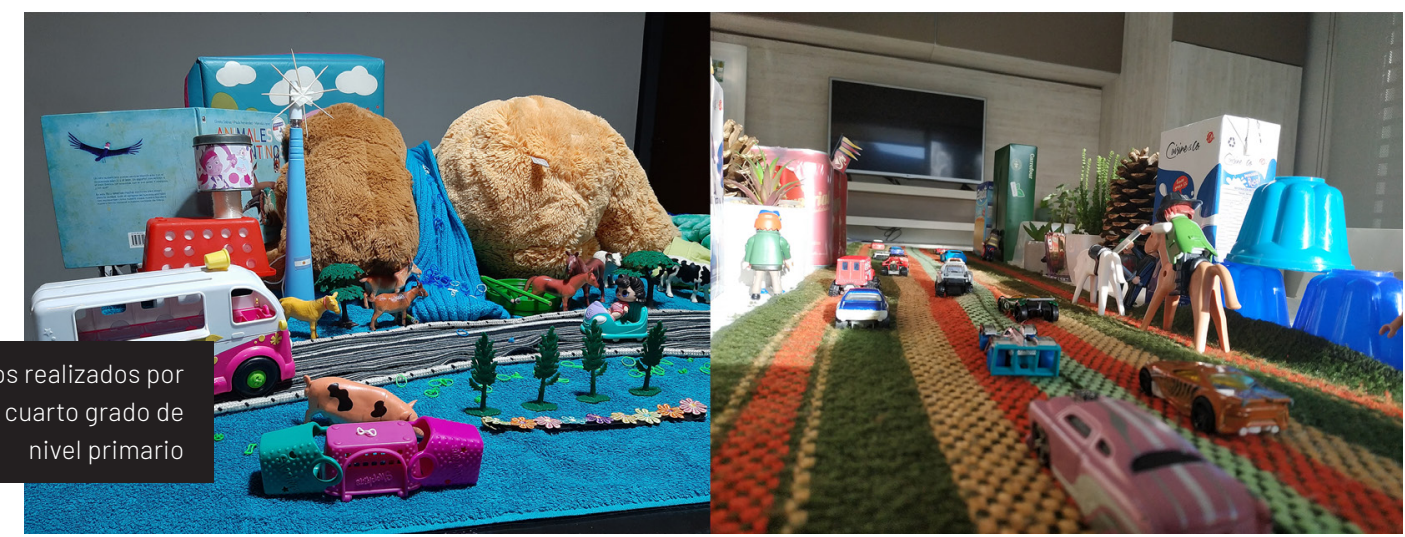

Comprendemos la experiencia desde la praxis, es decir, desde una relación dialéctica entre la práctica y la teoría, donde no hay una separación entre lo intelectual y lo manual(Belinche, 2017). «En las clases de Artes Visuales, el desarrollo de capacidades interpretativas pone el acento en la relación producción-reflexión, considerando la comprensión, la apropiación y la reelaboración crítica de la cultura» (Belardinelli \& Catibiela, 2017, p. 83). La experiencia, entonces, no solo involucra al momento de producción, sino también a las instancias de observación, de análisis y de reflexión, y a las preguntas que elaboramos para cada una de ellas, las cuales deberían estimular la curiosidad; a diferencia de las fácticas que esperan una respuesta unívoca:

En general, cuando hacemos preguntas que admitan como respuestas «sí» 0 «no», no obtenemos de nuestro interlocutor información suficiente para saber qué ha comprendido, qué está pensando o sintiendo. En lugar de estimular el diálogo, este tipo de preguntas cierran la posibilidad de argumentar ideas y de intercambiarlas (Anijovich \& Mora, 2010, p. 37).

Aunque durante el aislamiento se pierde la instancia de debate, de intercambio y de pensar las preguntas en conjunto, cuando las preguntas admiten la experiencia, promueven la interpretación y la construcción del conocimiento por parte de las y los estudiantes.

\section{¿Y ahora?}

En esta nueva normalidad o, mejor dicho, en este nuevo modo de estar siendo en el mundo nos enfrentamos a la incertidumbre, aunque tenemos la certeza de que la manera de hacerle frente es colectiva: "Nadie libera a nadie, ni nadie se libera solo. Los hombres se liberan en comunión» (Freire, [1968] 2013, p. 33). 
Es así, que en este contexto el arte promueve una lógica de pensamiento que puede aportar soluciones creativas ante la incertidumbre: tanto el modo de operar metafórico como el pensamiento divergente dejan de lado el pensamiento lineal y unívoco, con el fin de buscar múltiples resoluciones ante un problema. En este sentido, Hugo Zemelman (2006) explica la importancia de abordar múltiples lenguajes en la enseñanza, ya que ellos permiten anudarse al mundo y establecer relaciones más complejas con él. Así, posibilitan una mayor consciencia del mundo en el que vivimos y, en consecuencia, el desarrollo de la autonomía en la toma de decisiones y en el pensamiento.

Las y los docentes también estamos en un constante proceso de aprendizaje, en el cual la vigilancia epistemológica sobre la propia práctica se constituye como una herramienta fundamental: nos permite reflexionar sobre el sentido de la enseñanza a partir de las preguntas a quién enseñamos, qué enseñamos y cómo.

\section{Referencias}

Aisenberg, D. (2018). MDA. Apuntes para un aprendizaje del arte. Ciudad Autónoma de Buenos Aires, Argentina: Adriana Hidalgo editora.

Anijovich, R. (2016). Gestionar una escuela con aulas heterogéneas. Enseñar y aprender en la diversidad. Ciudad Autónoma de Buenos Aires, Argentina: Paidós.

Anijovich, R. y Mora, S. (2010). Estrategias de enseñanza: otra mirada al quehacer en el aula. Ciudad Autónoma de Buenos Aires, Argentina: Aique Grupo Editor.

Augustowsky, G. (2012). El arte en la enseñanza. Ciudad Autónoma de Buenos Aires, Argentina: Paidós.

Belardinelli, P. y Catibiela, A. (2017). Consideraciones sobre el arte y su enseñanza. Apuntes de clase. Metal, 3(3), 75-85. Recuperado de http://papelcosido.fba.unlp.edu.ar/ojs/index.php/ metal/article/view/462

Belinche, D. (2017). Diez formas de arruinar una clase. Recuperado de http://papelcosido.fba. unlp.edu.ar/libros/libro-diez-formas-arruinar.html

Canal ISEP. (23 de abril de 2020). "La clase en pantuflas». Conversatorio virtual con Inés Dussel. ISEP[Videoconferencia]. Disponible en https://www.youtube.com/watch?v=6xKvCtBC3Vs\&li st=LLA3bxMjNeqpVwpCUwWvYpUw\&index=3\&t=3s 
Consejo Federal de Educación. (25 de agosto de 2010). Resolución CFE N. ${ }^{\circ}$ 111/10 - Anexo. La educación artística en el sistema educativo nacional. Recuperado de http://repositorio. educacion.gov.ar:8080/dspace/bitstream/handle/123456789/54077/14862.pdf?sequence=1

Consejo Federal de Educación. (30 de septiembre de 2010). Resolución CFE N. ${ }^{\circ} 120 / 10$. Anexo 1. Criterios generales para la construcción de la Secundaria de Arte (Orientada, Especializada y Técnico-Artística). Recuperado de http://repositorio.educacion.gov.ar/dspace/bitstream/ handle/123456789/110044/ARt\%20resol\%20120\%20web.pdf?sequence=1

Consejo Federal de Educación. (15 de agosto de 2012). Resolución CFE N. ${ }^{\circ}$ 179/12. Anexos 1 a 5. Recuperado de http://repositorio.educacion.gov.ar:8080/dspace/bitstream/handle/123456789/110045/ARTE\%20Resol\%20179-192\%20web.pdf?sequence=1

Fba a la carta. (13 de mayo de 2020). Cuarentena, evaluación y después [Videoconferencia]. Disponible en https://www.youtube.com/watch?v=NK7Cyp75x2A\&t=854s

Freire, P. [1968](2013). Pedagogía del oprimido. Ciudad Autónoma de Buenos Aires, Argentina: Siglo Veintiuno.

Freire, P.(2006). El grito manso. Ciudad Autónoma de Buenos Aires, Argentina: Siglo Veintiuno.

Nicolás Trotta. (6 de mayo de 2020). Conferencia con Francesco Tonucci [Videoconferencia]. Disponible en https://www.youtube.com/watch?v=0Z5N-WjqKUA

Zemelman, H. (2006). El conocimiento como desafío posible. Ciudad de México, México: Instituto Politécnico Nacional, Instituto Pensamiento y Cultura en América Latina, A.C.

\section{Notas}

1 Usamos la palabra normalidad ya que es la que se escucha en este contexto, no obstante somos críticos ante la palabra normal y las significaciones que implica. 